\title{
All-Optical NRZ-to-RZ Format Conversion at 10 Gbit/s with 1-to-4 Wavelength Multicasting Exploiting Cross-Phase Modulation \& Four- Wave-Mixing in Single Dispersion-Flattened Highly Nonlinear Photonic Crystal Fiber
}

\author{
Zhan-Qiang Hui ${ }^{{ }^{*}}$, Bo Zhang ${ }^{1}$ and Jian-Guo Zhang ${ }^{2 *}$ \\ ${ }^{1}$ School of Electronic Engineering, Xi' an University of Posts and Telecommunications, Xi'an, 710121, China \\ ${ }^{2}$ School of Engineering, London South Bank University, 103 Borough Road, London SE1 0AA, UK \\ * zhanqianghui@xupt.edu.cn \\ jian-guo-zhang@126.com
}

\begin{abstract}
All-optical NRZ-to-RZ format conversion with a function of wavelength multicasting is proposed in this paper, which is realized by exploiting cross-phase modulation (XPM) and Four-Wave-Mixing (FWM) in a dispersion-flattened highly nonlinear photonic crystal fiber (DF-HNLPCF). The designed format converter is experimentally demonstrated, for which the 1-to-4 wavelength multicasting is achieved simultaneously by filtering out two FWM idler waves and both blue-chirped and red-chirped components of the broadened NRZ spectrum induced by XPM. Moreover, the wavelength tunability and dynamic characteristics of the proposed NRZto-RZ format converter are also exploited using the different central wavelengths of an optical clock signal and varying the input optical power at a DF-HNL-PCF in our experiment. It is shown that the designed format converter can possess a wide range of operational wavelength over $17 \mathrm{~nm}$, an optimal extinction ratio of $11.6 \mathrm{~dB}$ and a Q-factor of 7.1, respectively. Since the proposed scheme uses an optical-fiber-based configuration and is easy for implementation, it can be very useful for future applications in advanced fiber-optic communication networks.
\end{abstract}

Key words: Fiber-optic communications; Nonlinear optics; Four-wave mixing; Cross-phase modulation; Photonic crystal fiber

\section{Introduction}

With various new bandwidth-intensive services continuing to emerge, future advanced communication networks will use all-optical signal processing to eliminate the throughput bottleneck imposed by electrical-to-optical and optical-to-electrical conversions [1-3]. As a result, such networks can possess ultrahigh capacity and aggregate throughput to efficiently support terabit-per-second communication applications. To achieve this aim, dense wavelength-division multiplexing (WDM) should be adopted. In dense WDM (DWDM) networks, the non-return-to-zero (NRZ) data format is widely used due to its narrower spectral width and higher timing-jitter tolerance than employing the return-to-zero (RZ) format. On the other hand, it has been demonstrated that an improvement of optical receiver sensitivity by $2-4 \mathrm{~dB}$ could be achieved for RZ data signals compared with NRZ data [4]. This is due to the fact that the pulse energy is more confined near the center of the bit-slot for the RZ pulse signal, which can ultimately lead to increasing the transmission distance of an 
optical fiber link for a given bit-error rate. Moreover, RZ format has a larger tolerance to polarization mode dispersion (PMD), inter-symbol interference, self-phase modulation (SPM) and other long-haul fiber transmission impairments. Therefore, the established benefits of a $\mathrm{RZ}$ format have motivated the exploration of various techniques for all-optical format conversion of NRZ to RZ [4]. Until now, a number of methods have been proposed to achieve all-optical format conversion from NRZ to RZ signals by exploiting, for example

- Four-wave-mixing (FWM) [5], cross-phase modulation (XPM) [6], nonlinear polarization rotation [7] in a semiconductor optical amplifier (SOA);

- Cross-absorption modulation (XAM) in an electro-absorption modulator (EAM) [8];

- The cascaded second-harmonic generation and difference-frequency generation (cSHG/DFG) in a periodically poled lithium niobate (PPLN) waveguide [9];

- $\quad$ FWM in a passive GaAs bulk-waveguide [10];

- $\quad$ Either XPM or FWM in a silicon (Si) nanowire [11]

- By pulse carving of an NRZ optical data stream in a hybrid III-V-on-silicon technology platform [12];

- FWM [13-15], and optical Kerr switching \& pulse pre-chirping technique [16] in nonlinear optical fiber, Mach-Zehnder delay interferometer [17], respectively;

- Two-wavelength injection locked Fabry-Perot lasers [18].

All the schemes mentioned above have their individual advantages and respective drawbacks in terms of operation speed, wavelength range, manufacture cost, and system complexity of the resulting format converters. For example, the finite gain recovery time in SOA or EAM caused by the carrier lifetime can ultimately limit the maximum bit rate, while waveguide-based or nanowire-based format converters presently have a high cost and possess a high fiber-to-fiber insertion loss that can prevent them from practical applications nowadays. Delay interferometer, nonlinear optical loop mirror (NOLM) and injection locked Fabry-Perot laser have the complicated structure and are usually very sensitive to environmental parameter fluctuations.

The use of silica fibers as a nonlinear medium is more attractive due to their ultrafast nonlinear response. Nevertheless, the small nonlinear refractive index in traditional dispersion-shifted fibers (DSFs) makes a long interactive length or high input power necessary for obtaining sufficient nonlinear effects. To overcome this difficulty, a highly nonlinear fiber (HNLF) can be utilized to effectively shorten the fiber length or lower down input power required by optical format converters. This in turn makes HNLF-based format converters more attractive for practical applications in terms of manufacture cost and implementation complexity. However, the pulse walk-off and phase mismatch may limit the bit rate or wavelength flexibility in HNLF-based format converters. Recently, photonic crystal fibers (PCFs) have received significant attention for optical communications since they open new possibilities for the design and fabrication of optical fibers with tailorable optical properties [19]. Kwok et al. reported a 10Gbit/s NRZ-to-RZ format converter using XPM in a PCF [20]. However, the all-optical wavelength multicasting has not yet been considered in the work, while future photonic networks will employ all-optical multicasting to efficiently support bandwidth-intensive applications such as IP-TV, video distribution, teleconferencing and so on in DWDM networks.

In this paper, we experimentally demonstrate a tunable 10-Gbit/s NRZ-to-RZ format converter that consists of a single dispersion-flattened highly nonlinear PCF (DF-HNL-PCF) and four optical bandpass filters (OBPFs). The format conversion and one-to-four wavelength multicasting are based on XPM and FWM in the DF-HNL-PCF. This is achieved simultaneously by using four OBPFs to filter out the red-chirped and blue-chirped spectral components of the NRZ signal and two FWM-induced idler waves, respectively. Moreover, the wavelength tunability and dynamic characteristics of the proposed format converter are investigated by using an optical clock signal with different central wavelength and varying input optical power at a DF-HNL-PCF in our experiment. The results show that the newly designed format converter has a wide wavelength tuning range of $17 \mathrm{~nm}$ and a high tolerance 
to input optical power fluctuations. This property can make it more attractive than others for using in future ultrahigh-speed DWDM photonic networks.

\section{Operation principle}

Fig.1 shows the operation principle for all-optical format conversion from NRZ to RZ based on XPM and FWM in a DF-HNL-PCF. An optical clock-pulse stream at wavelength $\lambda_{\text {clock }}$ is combined with an optical data signal of NRZ format at wavelength $\lambda_{\mathrm{NRZ}}$ by a $2 \times 1$ optical power combiner. After passing through a high power erbium-doped fiber amplifier (HP-EDFA) for power amplification, they are launched into a DF-HNL-PCF. Here the HPEDFA is used to ensure that the powers of both NRZ data signal and clock-pulse stream are sufficiently high to create the XPM and FWM effects in the DF-HNL-PCF. In general, the injected clock pulses propagate in a nonlinear medium (i.e., DF-HNL-PCF) with high peak power and lead to a refractive-index change via the nonlinear index coefficient. This causes a chirp in the co-propagating NRZ data light via XPM and generates the sidebands on two sides of the NRZ data light. The leading edges of the NRZ data light are red-shifted, whereas the trailing edges are blue-shifted [21]. Consequently, the significant spectrum broadening of a NRZ signal light is observed at the output of a DF-HNL-PCF. Moreover, owing to the high nonlinearity and flattened dispersion of this DF-HNL-PCF, a FWM interaction between the optical clock pulse and the NRZ data signal occurs because the phase-matching condition can be easily satisfied. Then two idler waves are generated, which are distributed along both sides of the clock and NRZ signal wavelengths, marked as $\lambda_{\text {FWM-1 }}$ and $\lambda_{\text {FWM-2 }}$ in Fig. 1 , where $\lambda_{\text {FWM-1 }}$ $=1 /\left(2 / \lambda_{\text {Clock }}-1 / \lambda_{\mathrm{NRZ}}\right)$, and $\lambda_{\mathrm{FWM}-2}=1 /\left(2 / \lambda_{\mathrm{NRZ}}-1 / \lambda_{\text {Clock }}\right)$. The optical spectra evolution before and after the DF-HNL-PCF are also illustrated in Fig.1. Note that XPM leads to broadening the NRZ light spectrum. The output signal from a DF-HNL-PCF is divided into four parts via a $1 \times 4$ optical splitter (OS). Four sets of wavelength-tunable OBPFs are placed in parallel at the output of a $1 \times 4$ OS to select both blue- and red-chirped spectral components of a NRZ signal light as well as two FWM-generated idler waves at $\lambda_{\text {blue-chirped }}, \lambda_{\text {red-chirped }}, \lambda_{\text {FWM-1 }}$, and $\lambda_{\text {FWM-2 }}$, respectively.

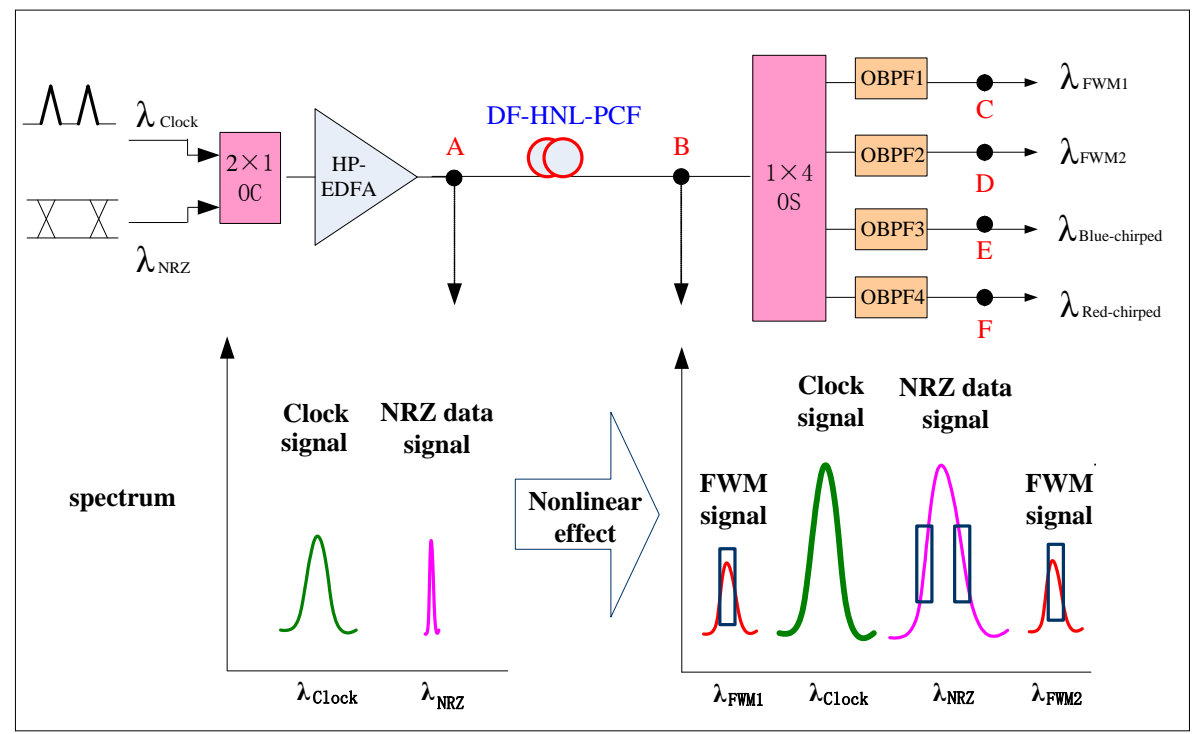

Figure 1. Operational principle of the NRZ-to-RZ format converter based on XPM and FWM in a DF-HNL-PCF. OC: optical coupler, HP-EDFA: high power erbium-doped fiber amplifier, OS: optical splitter, OBPF: optical bandpass filter. 


\section{Experimental setup}

The experimental setup of the designed all-optical NRZ-to-RZ format converter is illustrated in Fig.2. The continuous-wave (CW) light from a tunable laser (Santec, MLS-2100) is externally modulated by a Mach-Zehnder intensity modulator with $2^{31}-1$ pseudorandom binary sequence (PRBS) to generate a 10-Gbit/s optical NRZ data signal. A polarization controller (see Fig.2, PC1) is placed at the input port of the intensity modulator, so that the polarization state of the $\mathrm{CW}$ light is aligned with the transmission axis of the modulator in order to obtain the best modulation effect. An actively mode-locked semiconductor laser (AML-SL), which is synchronized with the PRBS generator (see Fig. 2), emits a $10-\mathrm{GHz}$ optical pulse train at the wavelength $\lambda_{\mathrm{AML}-\mathrm{SL}}=1550.31 \mathrm{~nm}$. The full width at half maximum (FWHM) of the generated pulse is approximately equal to $1.9 \mathrm{ps}$. After passing through a HPEDFA (HP-EDFA1, see Fig.2), the optical pulse train is injected into a 700-m dispersion shifted HNLF. The nonlinear coefficient of the HNLF is $9 \mathrm{~W}^{-1} \mathrm{~km}^{-1}$ and its dispersion is -2.42 $\mathrm{ps} /(\mathrm{nm} \mathrm{km})$ at $1550 \mathrm{~nm}$. The dispersion slope and attenuation coefficient of this HNLF is less than $0.02 \mathrm{ps} / \mathrm{nm}^{2} \mathrm{~km}$ and $0.43 \mathrm{~dB} / \mathrm{km}$ over a wavelength range from $1500 \mathrm{~nm}$ to $1600 \mathrm{~nm}$, respectively. The HP-EDFA1 is used to amplify the 10-GHz optical pulse train and ensure its power to be sufficiently high so as to create supercontinuum (SC) spectrum effect in the HNLF. At the output port of the HNLF, an OBPF (OBPF1, Santec: OTF-300) with bandwidth of $1.3 \mathrm{~nm}$ and tunable central wavelength is used to extract the desired SC spectral component. In doing so, now the obtained $10-\mathrm{GHz}$ optical pulse train at another wavelength of $\lambda_{\text {Clock }}$ serves as a $10-\mathrm{GHz}$ optical clock signal for the subsequent all-optical NRZ-to-RZ format conversion. After passing through two PCs (PC2 and PC3, see Fig.2) respectively, the NRZ optical data signal and control pulse train are combined by a $3 \mathrm{~dB}$ optical power coupler. Then they are launched into a 50-m DF-HNL-PCF (Crystal fiber, POS-1550) after optical amplification at another HP-EDFA (HP-EDFA2, see Fig.1). Note that HP-EDFA2 is required in the experiment to ensure the power of the combined optical clock and NRZ-data beams to be sufficiently high in order to create enough XPM and FWM effects in a DF-HNL-PCF. In practice, we can adjust PC2 and PC3 to align the polarization states of NRZ signal and clock lights so as to optimize the required nonlinear effects. Due to high nonlinear coefficient and flattened dispersion of the DF-HNL-PCF, the evident XPM and FWM effects occur. The nonlinear coefficient of the DF-HNL-PCF is $11 \mathrm{~W}^{-1} \mathrm{~km}^{-1}$ and its dispersion is $1.25 \mathrm{ps} /(\mathrm{nm}$ $\mathrm{km}$ ) with a variation $<0.25 \mathrm{ps} \mathrm{nm}^{-1} \mathrm{~km}^{-1}$ from $1500 \mathrm{~nm}$ to $1610 \mathrm{~nm}$, which in turn can ensure a wider operation wavelength range for the designed format converter. The attenuation coefficient of the DF-HNL-PCF is $9 \mathrm{~dB} / \mathrm{km}$ over $1500-1600 \mathrm{~nm}$. HP-EDFA1 and HP-EDFA2, which are commercially available, can provide a high saturation output power of $34 \mathrm{dBm}$ in the operation wavelength range from $1535 \mathrm{~nm}$ to $1565 \mathrm{~nm}$ and have a noise figure of $6 \mathrm{~dB}$. The output signal from a DF-HNL-PCF is divided into four parts via a $1 \times 4$ optical power splitter. Four sets of wavelength-tunable OBPFs (Santec, OTF-300) are employed in parallel at the output of a $1 \times 4$ optical splitter to select the blue- and red-chirped components of the broadened NRZ spectrum (induced by XPM) as well as two FWM-generated idler waves at $\lambda_{\text {blue-chirped }}, \lambda_{\text {red-chirped }}, \lambda_{\text {FWM-1 }}$, and $\lambda_{\text {FWM-2 }}$, respectively. The four OBPFs used for extracting multicasting signals are the same and have a Gaussian filtering profile with a $3 \mathrm{~dB}$ bandwidth of $0.38 \mathrm{~nm}$ and $20 \mathrm{~dB}$ bandwidth less than $1.2 \mathrm{~nm}$, thus being sufficiently wide for a $10 \mathrm{Gbit} / \mathrm{s}$ optical signal to pass through but a high roll-off rate outside the passband. In this case, the OBPFs can provide a sufficient rejection to the crosstalk from adjacent wavelength channel(s) and the optical noise from a HP-EDFA. Then the one-to-four wavelength multicasting of the format-converted optical data signal would be realized. The filtered RZ signal is injected into an optical receiver. The receiver is composed of an EDFA and an OBPF, followed by a 70 $\mathrm{GHz}$ photodetector $\left(\mathrm{U}^{2} \mathrm{t}\right.$ model number XPDV 3120R) for the optical-to-electrical conversion as well as a wide-bandwidth electrical digital sampling oscilloscope (Tektronix model number 80E06) of $70 \mathrm{GHz}$ for monitoring the waveforms and measuring the Q-factor of the formatconverted RZ data signal. The EDFA is used to amplify the power of the format-converted data signal from the DF-HNL-PCF to ensure that the sufficient photocurrent is fed into the oscilloscope. The OBPF6 with tunable bandwidth is used to suppress the amplified 
spontaneous emission noise of the EDFA. The Q-Factor represents the quality of the SNR in the "eye diagrams" of a digital signal. Generally speaking, the "eye diagrams" are the eyeshaped pattern on an oscilloscope, which can indicate the performance of a digital transmission system. The Q-factor was calculated according to the well-known expression $Q$ $=\left(P_{1}-P_{0}\right) /\left(\sigma_{1}-\sigma_{0}\right)$, where $P_{1}, P_{0}, \sigma_{1}$, and $\sigma_{0}$ are the average power and the standard deviation on "ones" and "zeros," respectively. They are usually obtained by using a digital sampling oscilloscope to measure both voltage histograms at the center of the eye diagram and the standard deviation of the noise at both signal levels [22]. To monitor the optical spectra of clock signal, NRZ signal and the converted RZ signals on different multicasting channels, we also connect an optical spectrum analyzer (OSA, Yokogawa-AQ6370) of resolution $0.02 \mathrm{~nm}$ with the input and output ports of the HNLF, the output port of the OBPF1, the input and output ports of the DF-HNL-PCF, and the output port of the OBPF2, OBPF3, OBPF4, OBPF5, corresponding to points A, B, C, D, E, F, G, H and I as shown in Fig.2.

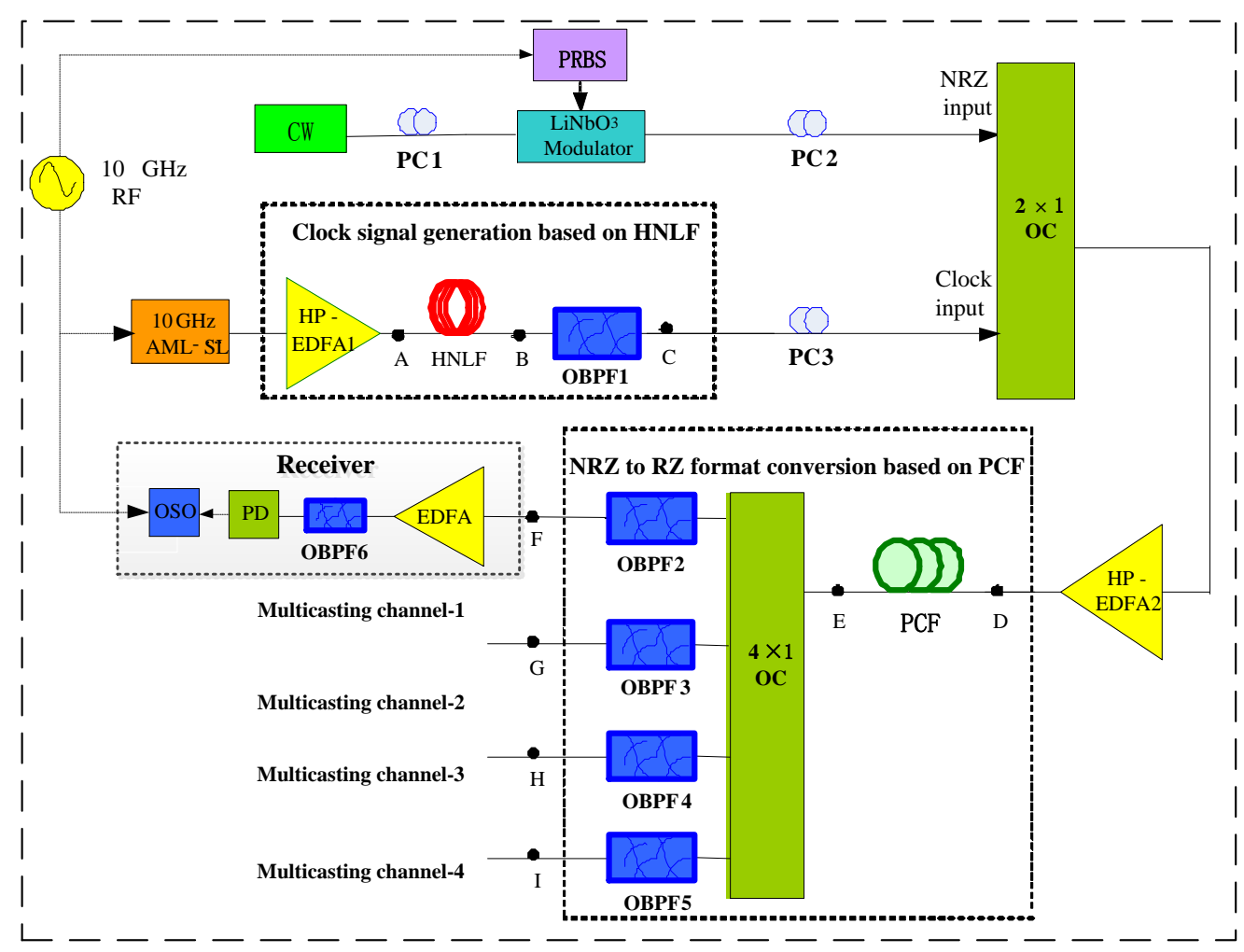

Figure 2. Experimental setup of the proposed format converter with 1-to-4 wavelength multicasting. AML-SL: actively mode-locked semiconductor laser; PC: polarization controller; PRBS: pseudorandom binary sequences; EDFA: erbium-doped fiber amplifier; OBPF: optical band-pass filter; HP-EDFA: high power erbium-doped fiber amplifier; OSO: oscilloscope; PD: photodetector; PCF: photonic crystal fiber.

\section{Results}

The proposed NRZ-to-RZ format conversion scheme mainly consists of two functional sections, i.e., clock signal generation and NRZ-to-RZ format conversion, respectively. The wavelength of the NRZ signal light is $\lambda_{\mathrm{NRZ}}=1550 \mathrm{~nm}$, while the wavelength of the original optical pulse train from an AML-SL is $\lambda_{\mathrm{AML}-\mathrm{SL}}=1550.31 \mathrm{~nm}$. To achieve all-optical NRZ-to- 
RZ format conversion, a synchronous optical clock signal with high extinction ratio (ER) and low timing jitter is required. Furthermore, the wavelengths of both clock and NRZ-signal lights should be distinct from each other so as to achieve the favorable results for NRZ-to-RZ format conversion. Based on practical experimental condition at our laboratory, an optical clock signal can be obtained through spectrum slicing of the SC spectrum obtained from the output port of a 700-m HNLF. The SC spectrum generation in optical fiber is a rich and complex process that involves various nonlinear effects. The combination of these nonlinear effects results in the emission with extremely broad spectra [23]. This process has been implemented in clock signal generation section, which has an advantage of eliminating the use of any other pump source. In our experiment, the total power of an optical signal that is launched into the HNLF for generating a SC spectrum is equal to $27.5 \mathrm{dBm}$. The optical spectrum of an original pulse signal, the SC spectrum after passing through the 700-m HNLF, and the sliced spectrum after OBPF1 are measured and shown in Fig. 3, corresponding to points A, B, and C as illustrated in Fig. 2, respectively. It can be seen that a severe spectral broadening with flattened top occurs at the HNLF output due to high fiber nonlinear coefficient and intense input pulse power. The $20-\mathrm{dB}$ spectrum width is about $36 \mathrm{~nm}$. A wavelength-tunable OBPF with central wavelength chosen at $1545 \mathrm{~nm}$ is used to extract the desired spectral component so as to produce the required optical clock signal. The eye diagrams of original NRZ data signal and the obtained $10-\mathrm{GHz}$ clock pulse signal are recorded and shown in Fig. 4.

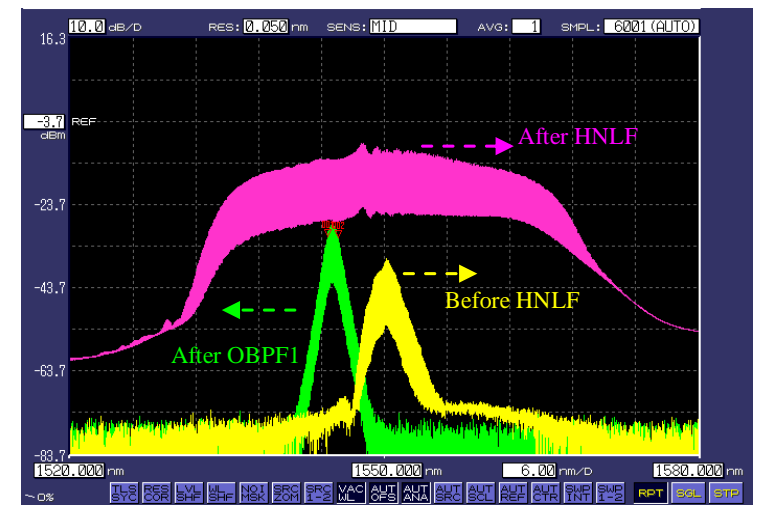

Figure 3. The measured optical spectra before HNLF, after HNLF, and after OBPF1
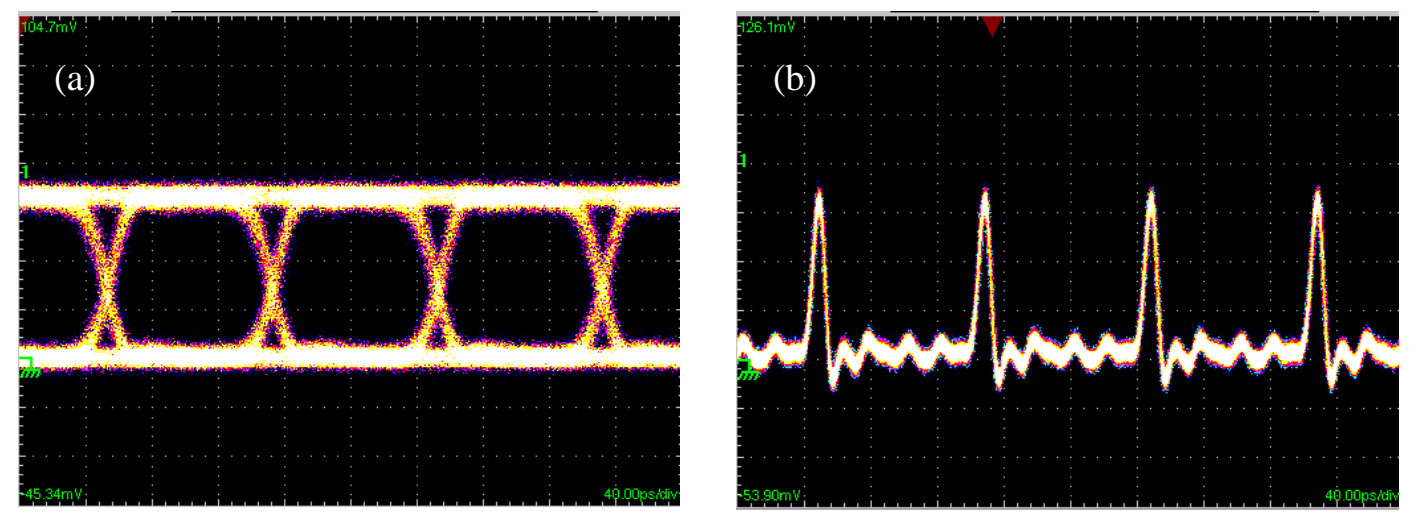

Figure 4. Eye diagrams of (a) original NRZ signal and (b) clock control light pulse. 
Both optical NRZ data signal and clock pulse train are then coupled into another HPEDFA (HP-EDFA2, see Figure 2) through a 3-dB optical coupler. After power amplification, the combined lights are injected into a 50-m DF-HNL-PCF. The two polarization controllers are used to align the polarization states of both NRZ-signal and clock-pulse lights in order to optimize the XPM and FWM effects between them. Fig. 5 shows the optical spectra of NRZ signal and control light measured by an OSA before entering the PCF, after passing through the PCF, and after OBPF2, OBPF3, OBPF4 and OBPF5 (corresponding to points D, E, F, G, $\mathrm{H}$ and I respectively, see Fig.2), respectively. The $20 \mathrm{~dB}$ spectrum width of the NRZ data signal is broadened obviously, which is caused by XPM between the pump clock pulse and optical NRZ signal. Moreover, the evident new frequency spectral components induced by FWM between NRZ-signal and clock control lights are also generated, which can be attributed to the stronger power of input optical signals and the flattened dispersion of a DFHNL-PCF. The output light from a DF-HNL-PCF is divided into four parts, the subsequent optical filters (i.e., OBPF2, OBPF3, OBPF4 and OBPF5) are used to filter out the bluechirped and red-chirped components of the broadened NRZ-signal spectrum and two FWMgenerated new spectral components at $\lambda_{\text {blue-chirped }}, \lambda_{\text {red-chirped }}, \lambda_{\text {FWM-1 }}$, and $\lambda_{\text {FWM-2 }}$ respectively. The central wavelengths of the four OBPFs are $1549.25 \mathrm{~nm}, 1550.70 \mathrm{~nm}, 1540.21 \mathrm{~nm}$, and $1554.78 \mathrm{~nm}$, respectively. Then the four converted RZ optical signals are obtained from the outputs of those OBPFs.

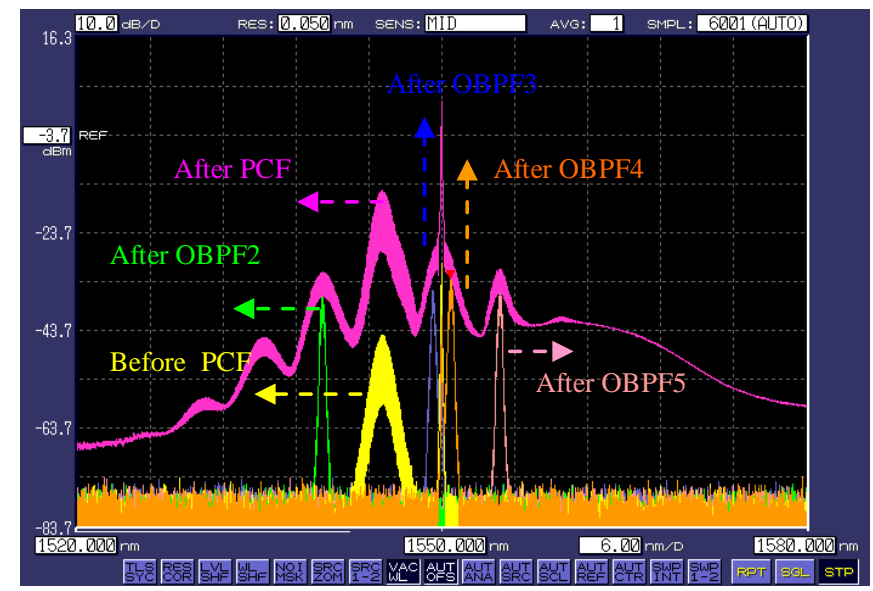

Figure 5. The optical spectra before PCF, after PCF, after OBPF2, OBPF3, OBPF4 and OBPF5, respectively.

Fig. 6 shows the recorded eye diagrams of the format-converted RZ signals at $10 \mathrm{Gbit} / \mathrm{s}$ on four wavelength-multicasting channels, as mentioned above. It can be seen that the clear and widely open eye diagrams with the same bit rate as an original NRZ data signal are observed for the four converted RZ data signals. This means that the one-to-four wavelength multicasting is achieved together with the optical NRZ-to-RZ format conversion. The original NRZ signal has a Q-factor of 13.4 and the ER of $20 \mathrm{~dB}$. The measured Q-factors of the converted $\mathrm{RZ}_{1}$ (left sideband), $\mathrm{RZ}_{2}$ (right sideband), $\mathrm{RZ}_{3}$ (FWM1), and $\mathrm{RZ}_{4}$ (FWM2) are 6.2, 6.6, 5.5, and 5.8, respectively. The output ERs of $\mathrm{RZ}_{1}, \mathrm{RZ}_{2}, \mathrm{RZ}_{3}$, and $\mathrm{RZ}_{4}$ are $11.2 \mathrm{~dB}, 11.3$ $\mathrm{dB}, 9.5 \mathrm{~dB}$, and $9.8 \mathrm{~dB}$, respectively. Therefore, the ER degradation is about $8.8 \mathrm{~dB}, 8.7 \mathrm{~dB}$, $10.5 \mathrm{~dB}$, and $10.2 \mathrm{~dB}$, respectively, while the Q-factor degradation is also evident. The degradation of the converted RZ signals can be attributed to the timing jitter of the original ultra-short pulse train from the actively mode-locked laser and the amplified spontaneous emission noise of HP-EDFAs. 

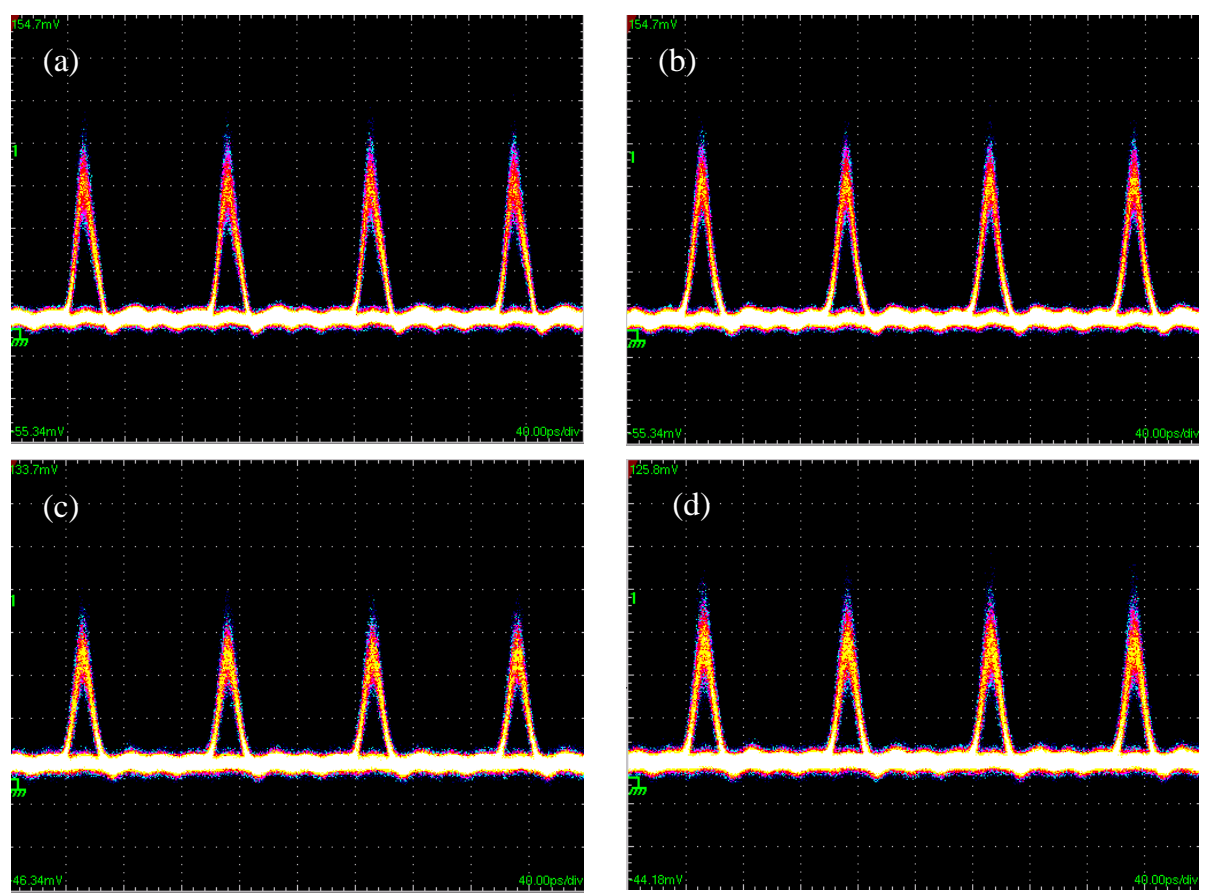

Figure 6. Eye diagrams of the converted RZ signal for (a) the blue-shifted sideband $\left(R Z_{1}\right)$ at $\lambda=1549.25 \mathrm{~nm}$, (b) the red-shifted sideband $\left(\mathrm{RZ}_{2}\right)$ at $\lambda=1550.70 \mathrm{~nm}$, (c) FWM1 $\left(\mathrm{RZ}_{3}\right)$ at $\lambda=1540.21 \mathrm{~nm}$, and (d) FWM2 $\left(\mathrm{RZ}_{4}\right)$ at $\lambda=1554.78 \mathrm{~nm}$, respectively.

\section{Discussions}

\subsection{Wavelength tunability}

As far as all-optical NRZ-to-RZ format converter is concerned, a wide operating wavelength range is highly desirable for practical applications, which can improve the capacity and flexibility of DWDM photonic networks and can also reduce the management cost. Therefore, we carry out an experimental investigation into the wavelength tuning range of the designed format converter. In this test, the $10 \mathrm{Gbit} / \mathrm{s}$ optical NRZ data signal has a fixed central wavelength of $\lambda_{\mathrm{NRZ}}=1550 \mathrm{~nm}$, whereas the clock signal with a different central wavelength of $\lambda_{\text {Clock }}$ is obtained by means of slicing the SC spectrum in the clock signal generation section. The tuning characteristic of an optical NRZ-to-RZ format converter is measured by varying the central wavelength of an optical clock signal. In principle, the resulting SC spectrum covers the whole conventional wavelength band (i.e., C-band) of 36 $\mathrm{nm}$, which enables us to obtain the optical clock signal at an arbitrary central wavelength within the C-band. For convenience, in our experiment the obtained optical clock signals are demonstrated only at several selected wavelengths. This is done by using a tunable OBPF with 1.3-nm bandwidth to select the different spectral components of a SC spectrum at the specific wavelengths of $1543 \mathrm{~nm}, 1555.62 \mathrm{~nm}, 1558.45 \mathrm{~nm}$, and $1560.65 \mathrm{~nm}$, respectively. Fig. 7 shows the corresponding optical spectra recorded at points A, B, C, and D (see Fig. 2), respectively. The corresponding eye diagrams of $10-\mathrm{GHz}$ optical clock pulse obtained from four different wavelengths are recorded and shown in Figure 8. It shows that the obtained four clock signals have a high ER. They are suitable for use in the following test of all-optical NRZ-to-RZ format conversion. 

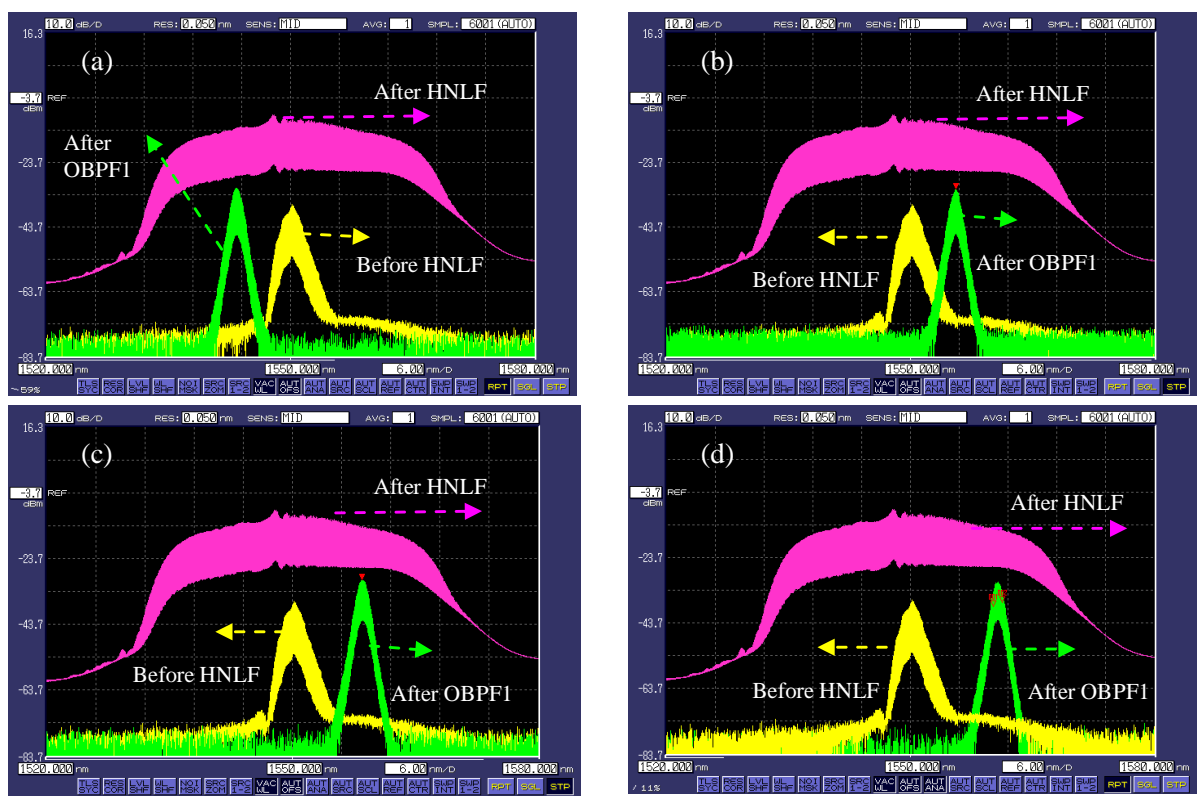

Figure 7. The optical spectra before HNLF, after HNLF, after OBPF1 for the generation of an optical clock signal at the wavelength (a) $\lambda_{\text {Clock }}=1543 \mathrm{~nm}$, (b) $\lambda_{\text {Clock }}=1555.62 \mathrm{~nm}$, (c) $\lambda_{\text {Clock }}=1558.45 \mathrm{~nm}$, and (d) $\lambda_{\text {Clock }}=1560.65 \mathrm{~nm}$, respectively.

To perform the optical NRZ-to-RZ format conversion, both the NRZ-data and clockpulse lights with different central wavelengths are launched into a 50-m DF-HNL-PCF. Fig. 9 shows the optical spectra of both NRZ signal and clock signal measured by an OSA before entering the PCF, after passing through the PCF, and after the OBPF2, OBPF3, OBPF4 and OBPF5 (corresponding at points D, E, F, G, H, and I in Fig.2, respectively), respectively. As can be seen, the 20-dB spectral width of the NRZ signal light is broadened. Moreover, FWMinduced new frequency components also appear. All of these can be attributed to the XPM and FWM effects between the NRZ signal and clock signal because of a stronger input optical power and the flattened dispersion of the DF-HNL-PCF. The four converted RZ signals with clear eye diagrams are obtained when the four clock signals with different central wavelengths are used in the experiment. The results indicate that our designed all-optical format converter can achieve a wide operation wavelength range over nearly $17 \mathrm{~nm}$. This is substantially limited by the operation wavelength range of the OBPF and HP-EDFA in our experiments. Furthermore, the operation of the designed all-optical format converter is transparent to the bit rate since it has the potential of attaining a terabit-per-second operation speed because of using the ultra-fast third-order optical nonlinearity in an optical fiber. 


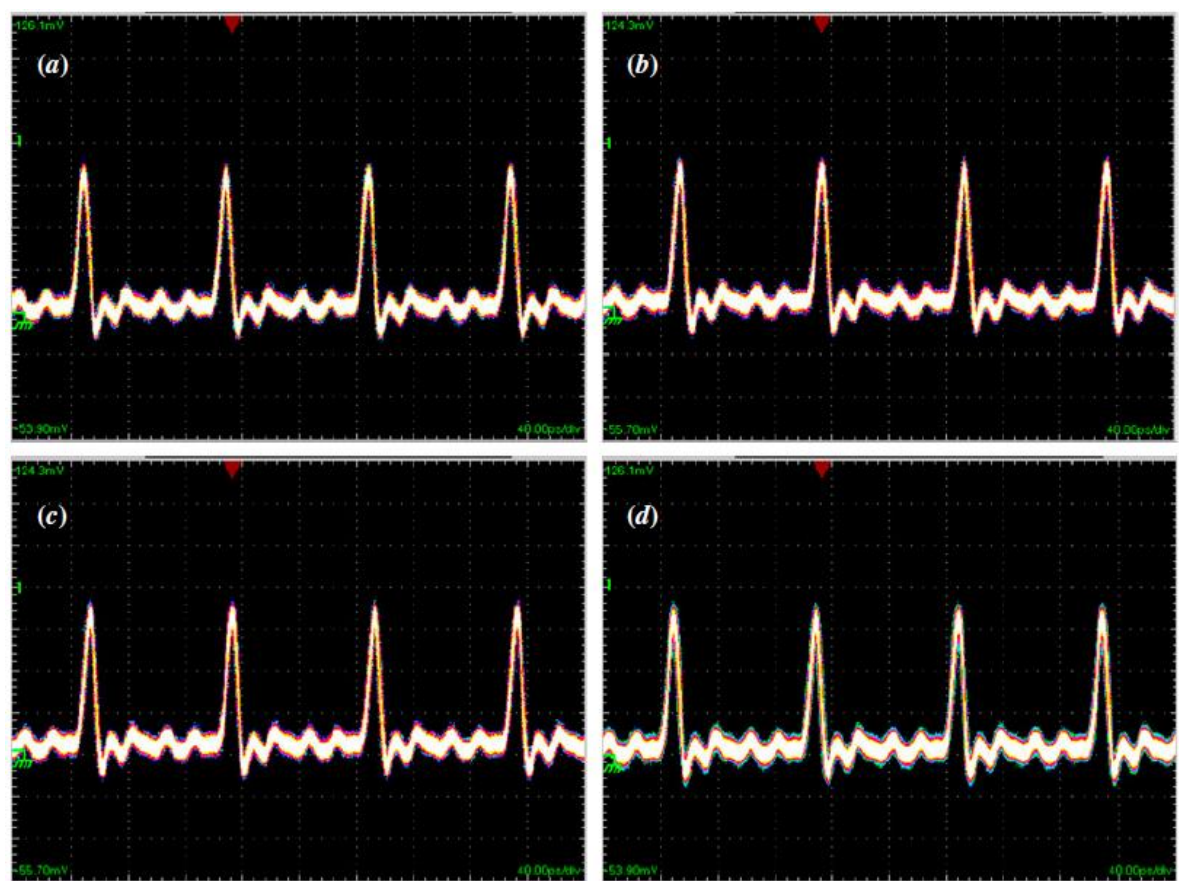

Figure 8. Eye diagrams of the optical clock pulse at the wavelength of (a) $\lambda_{\text {Clock }}=1543 \mathrm{~nm}$, (b) $\lambda_{\text {Clock }}=1555.62 \mathrm{~nm}$, (c) $\lambda_{\text {Clock }}=1558.45 \mathrm{~nm}$, and (d) $\lambda_{\text {clock }}=1560.65 \mathrm{~nm}$, respectively.
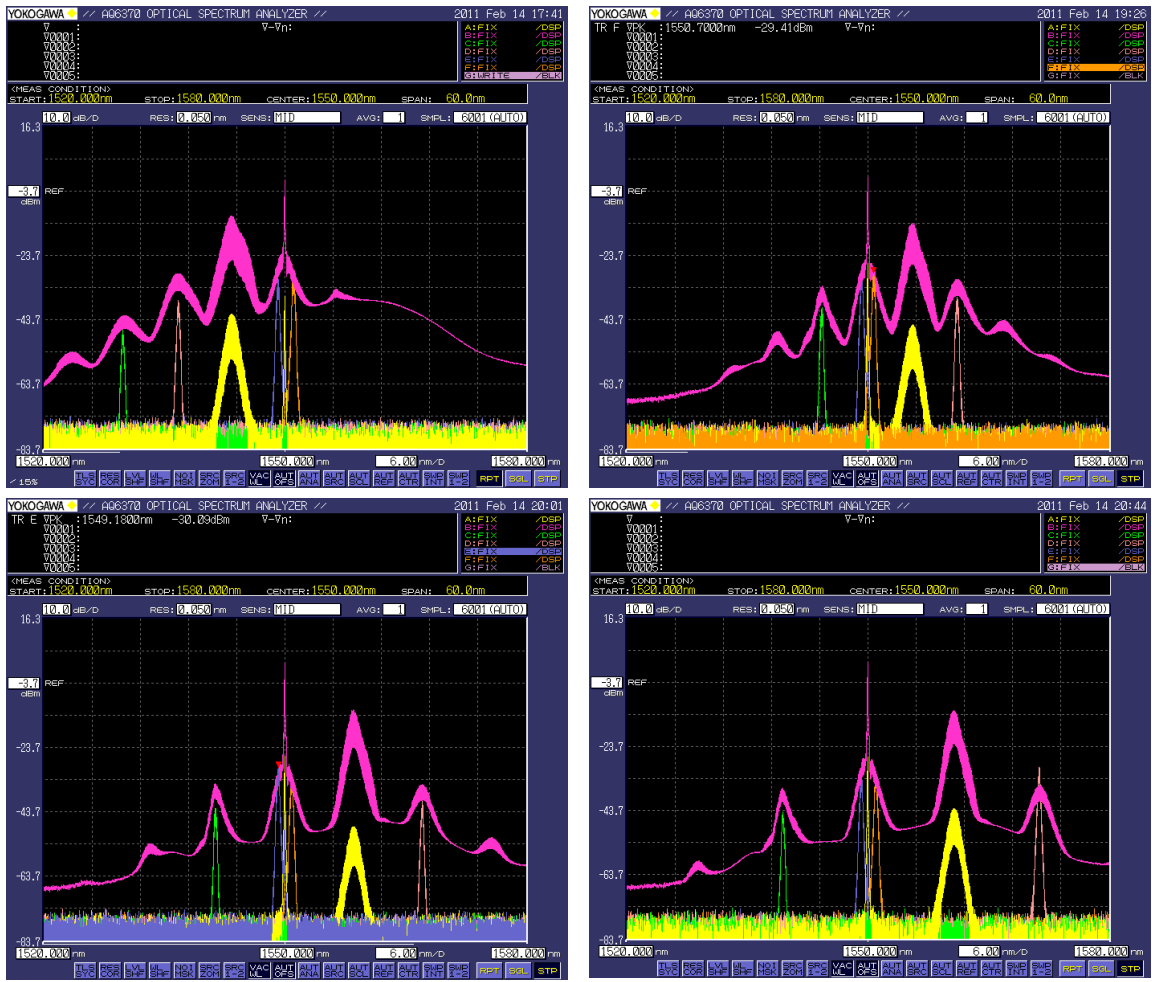

Figure 9. The optical spectra before PCF, after PCF, after OBPF2, OBPF3, OBPF4 and OBPF5 for optical NRZ-toRZ format conversion with wavelength of a clock light at (a) $\lambda_{\text {Clock }}=1543 \mathrm{~nm}$, (b) $\lambda_{\text {Clock }}=1555.62 \mathrm{~nm}$, (c) $\lambda_{\text {Clock }}=1558.45 \mathrm{~nm}$, and (d) $\lambda_{\text {Clock }}=1560.65 \mathrm{~nm}$. 


\subsection{Power tolerance}

For practical optical fiber communication applications, the optical power variation cannot be avoided completely due to the random change of the system operation environment/conditions. This requires the all-optical NRZ-to-RZ format converter to have some tolerance to the fluctuation of input optical power. It can become an important issue on the design of all-optical format converters. To facilitate the practical design and engineering applications, we have carried out an investigation into the impact of input signal power on the $\mathrm{Q}$ factor and ER of the four converted RZ optical signals. In our test, the wavelengths of NRZ data and clock lights are 1550 and $1555.62 \mathrm{~nm}$, respectively, while the length of DF-HNLPCF remains $50 \mathrm{~m}$ as before. The input powers are set as 22.5 to $27.5 \mathrm{dBm}$ in a step of $1 \mathrm{~dB}$ by adjusting the pump current of HP-EDFA. The other parameters are constant to make the results comparable. Fig. 10 indicates the evolution of output optical spectra for FWM-induced idler waves and the XPM-induced NRZ spectrum broadening, respectively, with the increase of the input optical power. The spectrum before DF-HNL-PCF is also shown in Fig. 10 for comparison. It can be seen that, when the input power is increased, the NRZ spectrum broadening induced by XPM becomes more noticeable, and the power level of the two idler waves is also increased. The Q factor and ER of four converted RZ signals are measured against the input optical power for a DF-HNL-PCF based format converter, and the obtained results are shown in Fig. 11. It can be seen that the maximum $Q$ factor of 7.1 and the best ER of $11.6 \mathrm{~dB}$ are obtained when the input optical power is $27.5 \mathrm{dBm}$. However, the degradation of $\mathrm{Q}$ factor and ER becomes rapid when the input power is lower than $24.5 \mathrm{dBm}$ in our measurement range. Note that the optical power higher than $27.5 \mathrm{dBm}$ is beyond the scope of our investigation due to the limited power tolerance of commercially available OBPF at our laboratory. From Fig. 11, we can see that the Q factor is larger than 4 and the ER is higher than $5 \mathrm{~dB}$ for the input optical power ranging from $24.5 \mathrm{dBm}$ to $27.5 \mathrm{dBm}$. The performance is acceptable within this range of 3-dB power variation. It shows that the proposed format converter has some tolerance to the optical power fluctuation. This property can be useful for the engineering design and applications of PCF-based NRZ-to-RZ format converters, because it can alleviate the strict requirement of controlling the optical power.

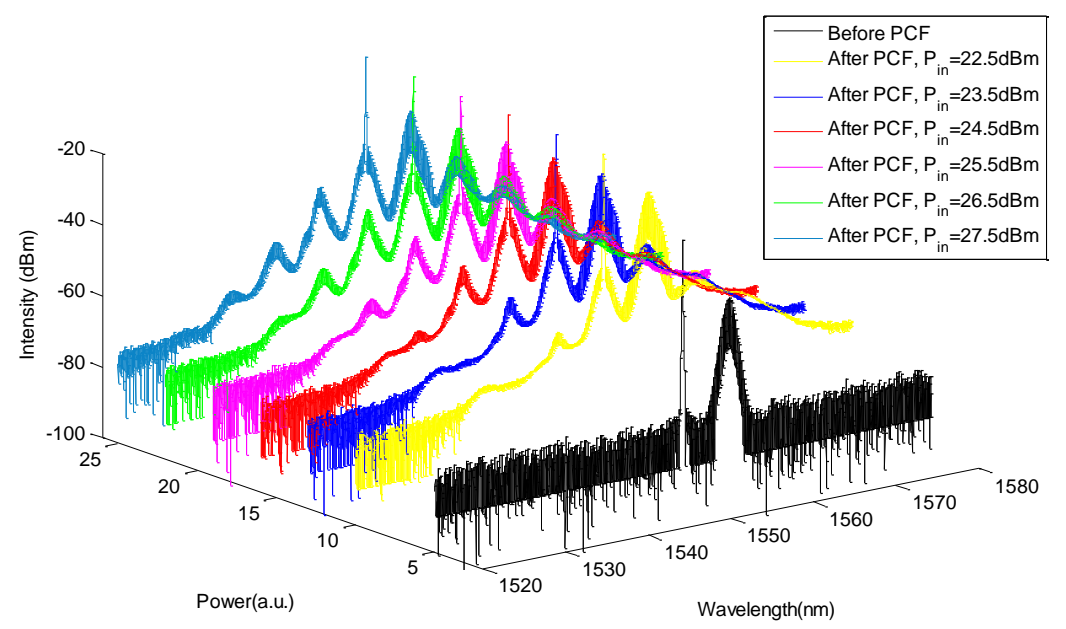

Figure 10. The output optical spectra from the DF-HNL-PCF when $\lambda_{\mathrm{NRZ}}=1550 \mathrm{~nm}$ and $\lambda_{\text {clock }}=1555.62 \mathrm{~nm}$, for different input powers. 

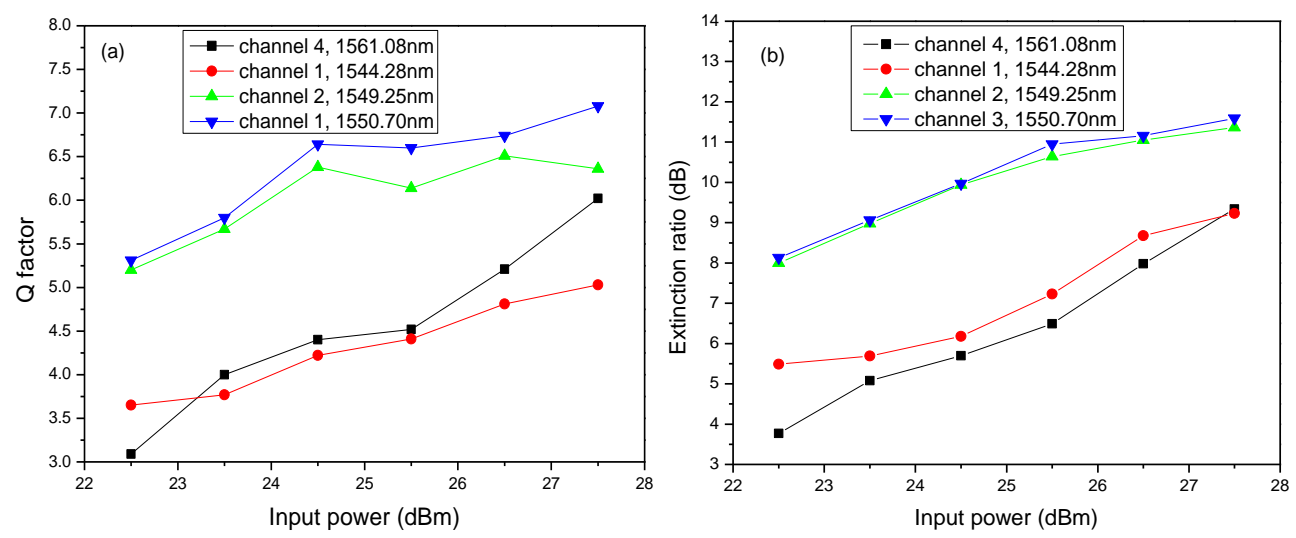

Figure 11. The measured results of (a) Q-factor and (b) ER of the converted RZ signals on multicasting channels 1,2, 3 and 4 under the condition of different input powers.

\section{Conclusions}

In this work, we have presented the effective design of a widely tunable NRZ-to-RZ format converter with one-to-four wavelength multicasting capability. The proposed format converter consists of a single 50-m DF-HNL-PCF and four OBPFs. By exploiting XPM and FWM effects in the DF-HNL-PCF, the 10-Gbit/s RZ signal on four different central wavelength channels is obtained by simultaneous filtering of both blue-chirped and red-chirped components of the broadened NRZ spectrum induced by XPM as well as two spectral components of FWM-induced idle waves, respectively. The wavelength tunability has been demonstrated by utilizing an optical clock signal with a different central wavelength by SC spectrum slicing. Furthermore, the impact of the input optical power variation on the quality of converted RZ signals for the designed format converter is also investigated. The results show that the designed format converter has a wide range of operational wavelength over 17 $\mathrm{nm}$, an optimal ER of $11.6 \mathrm{~dB}$ and a $\mathrm{Q}$ factor of 7.1, respectively. The proposed scheme is simple for implementation and uses an optical-fiber-based configuration to support ultrafast operations, thus making it attractive for use in future advanced optical fiber communication networks.

\section{Acknowledgements}

This work is supported by the National Nature Science Fund of China (Number: 61201193), the National High Technology Research and Development Program of China (Number: 2013AA014504), the Science and Technology Attack Project in Shaanxi Province (No. 2014k05-40), the Foundation of Shaanxi Educational Committee (Number: 2013JK1094), and the Xi'an Municipal Science and Technology Bureau Scientific Research Program (Number: CXY1436), respectively. 


\section{References}

1. C. Langrock; S. Kumar; J. E. McGeehan; A. E. Willner; and M. M. Fejer. J. Lightw. Technol. 2006, 24, 2579-2592.

2. C.H. Kwok; S.H. Lee; K.K. Chow; C. Lin. IET Optoelectron. 2007, 1, 47-53.

3. D. Norte; A. E. Willner; IEEE Photon. Technol. Lett. 1995, 7, 1354-1356.

4. W. Astar, Jeffrey B. Driscoll, Xiaoping Liu, Jerry I. Dadap, William M. J. Green, Yurii A. Vlasov, Gary M. Carter, and Richard M. Osgood, Jr. Optics Express. 2009, 17, 1298712999.

5. Andrea Reale; Paolo Lugli; Silvello Betti. Journal on Selected Topics in Quantum Electronics. 2001, 17, 703-709.

6. X. Yang; A.K. Mishra; R.J. Manning; R.P. Webb; A.D. Ellis. Electronics Letters. 2007, 43, 890-892.

7. X. Yang; A.K. Mishra; R.J. Manning; R. Giller. Electronics Letters. 2007, 43, 469-471.

8. Zhixin Chen; Jian Wu; Kun Xu. Optical Engineering. 2007, 46, 080502.

9. Jian Wang; Junqiang Sun; Qizhen Sun; Dalin Wang; Minjuan Zhou. Optics Letters. 2007, 32, 2462-2464.

10. Paveen Apiratikul; W. Astar; Gary M. Carter; Thomas E. Murphy. IEEE Photonics Technology Letters. 2010, 22, 872-874.

11. W. Astar; Jeffrey B. Driscoll; Xiaoping Liu; Jerry I. Dadap; William M. J. Green, Yurii A. Vlasov. IEEE Journal on Selected Topics in Quantum Electronics. 2010, 16, 234-249.

12. R. Kumar; T. Spuesens; P. Mechet; N. Olivier; J.-M. Fedeli; P. Regreny; G. Roelkens. Optics Express 2011, 19, 24647-24656.

13. H. Zhan-Qiang; Z. Jian-Guo. Acta Phys. Sin. 2013, 62, 084209.

14. E.J.M. Verdurmen; G.D. Khoe; A.M.J. Koonen; H. de Waardt. Microwave and Optical Technology Letters 2006, 48, 992-994.

15. L.-S. Yan; A.-L. Yi; W. Pan; B. Luo; J. Ye. Optics Express 2010, 18, 21404-21409.

16. Bill Ping-Piu Kuo; P. C. Chui; Kenneth Kin-Yip Wong. IEEE Journal of Lightwave Technology. 2008, 26, 3770-3775.

17. Jianjun Yu; Gee Kung Chang, John Barry; Yikai Su. Optics Communications. 2005, 248, 419-422.

18. C.W. Chow; C.S. Wong. Electronics Letters. 2003, 39, 997-999.

19. P. Russell. Science 2003, 299, 358-362.

20. C. H. Kwok; Chinlon Lin. IEEE Journal on Selected Topics in Quantum Electronics. 2006, $12,451-458$.

21. Zhanqiang Hui; Jian-Guo Zhang. Journal of Optics. 2012, 14, 055402.

22. S. Mikroulis, A. Bogris, E. Roditi, and D. Syvridis. Journal of Lightwave Technology. 2004, 22, 2743-2748.

23. Karen Marie Hilligsøe; Thomas Vestergaard Andersen; Henrik Nørgaard Paulsen; Carsten Krogh Nielsen; Klaus Mølmer. Optics Express. 2004, 12, 1045-1054. 